Supporting Information

\title{
Conductivity and Stability Enhancement of PEDOT:PSS Electrodes via Facile Doping of Sodium 3-Methylsalicylate for Highly Efficient Flexible Organic Light-Emitting Diodes
}

Lihui Liu,,$^{\dagger}$ Lei Wu,,$^{\dagger}$ Hao Yang, ${ }^{\dagger}$ Honggang Ge,,$^{\dagger}$ Juxuan Xie,,$^{\dagger}$ Kun Cao, ${ }^{\dagger}$ Gang Cheng ${ }^{\ddagger}, \#$ and Shufen Chen $^{\dagger, *}$

†State Key Laboratory for Organic Electronics and Information Displays \& Institute of Advanced Materials (IAM), Nanjing University of Posts \& Telecommunications, Nanjing 210023, China

Hong Kong Quantum AI Lab Limited, 17 Science Park West Avenue, Pak Shek Kok, Hong Kong SAR, China

${ }^{*}$ HKU Shenzhen Institute of Research and Innovation, Shenzhen 518053, China

* Corresponding Author

E-mail address: iamsfchen@njupt.edu.cn. 
Table S1 Device performance of OLEDs with PEDOT:PSS electrodes. (G: green light-emitting OLEDs; R: red light-emitting OLEDs; B: blue light-emitting OLEDs; W: white light-emitting OLEDs)

\begin{tabular}{|c|c|c|c|c|c|}
\hline Year Ref. & $\begin{array}{c}\sigma(\mathrm{s} / \mathrm{cm}) \text { or } \\
\mathrm{R}_{\mathrm{s}}(\Omega / \square)\end{array}$ & Device structure & $\begin{array}{c}\mathbf{C E} \\
(\mathbf{c d} / \mathrm{A})\end{array}$ & $\begin{array}{c}\mathbf{P E} \\
(\operatorname{lm} / \mathbf{W})\end{array}$ & $\begin{array}{l}\text { EQE } \\
(\%)\end{array}$ \\
\hline $2002^{1}$ & $1850 \Omega / \square$ & PEDOT:PSS electrode/TPD/Alq $3 / \mathrm{Mg}: \mathrm{Ag}$ & & & $\begin{array}{l}0.73 \\
(\mathrm{G})\end{array}$ \\
\hline $2007^{2}$ & $500 \mathrm{~s} / \mathrm{cm}$ & $\begin{array}{c}\text { PEDOT:PSS } \\
\text { electrode/MeO-TPD:F }{ }_{4}-\mathrm{TCNQ} / \text { Spiro-TAD/ } \\
\text { emission layer/TPBi/BPhen:Cs/Al }\end{array}$ & $\begin{array}{c}13.2 \\
(\mathrm{R}) / 62.0 \\
(\mathrm{G}) / 4.2 \\
(\mathrm{~B})\end{array}$ & $\begin{array}{c}15.9 \\
(\mathrm{R}) / 63.5 \\
(\mathrm{G}) / 4.0 \\
\text { (B) }\end{array}$ & \\
\hline $2008^{3}$ & $300 \mathrm{~s} / \mathrm{cm}$ & PEDOT:PSS electrode/NPB/Alq $/ 3 / \mathrm{LiF} / \mathrm{Al}$ & $2.23(\mathrm{G})$ & & \\
\hline $2012^{4}$ & $227 \Omega / \square$ & $\begin{array}{l}\text { Glass/PEDOT:PSS electrode/ } \mathrm{MoO}_{3} \\
\text { /NPD/CBP:Ir(ppy) })_{3} / \mathrm{BPhen} / \mathrm{LiF} / \mathrm{Al}\end{array}$ & $127(\mathrm{G})$ & $118(\mathrm{G})$ & $\begin{array}{l}40 \\
(\mathrm{G})\end{array}$ \\
\hline $2013^{5}$ & $55 \Omega / \square$ & PEDOT:PSS electrode/NPB/Alq $/ 3 / \mathrm{LiF} / \mathrm{Al}$ & & $1.6(\mathrm{G})$ & $\begin{array}{l}1.37 \\
(\mathrm{G})\end{array}$ \\
\hline $2013^{6}$ & $228 \Omega / \square$ & $\begin{array}{l}\text { SLG-TiOx-PEDOT:PSS/NPB/ } \\
\qquad \mathrm{Alq}_{3}: \mathrm{C} 545 \mathrm{~T} / \mathrm{Alq}_{3} / \mathrm{LiF} / \mathrm{Al}\end{array}$ & $10.11(\mathrm{G})$ & $5.41(\mathrm{G})$ & \\
\hline $2014^{7}$ & $800 \mathrm{~s} / \mathrm{cm}$ & $\begin{array}{l}\text { PEDOT:PSS electrode/NPB/Alq } / \\
\text { Bphen/Bphen: } \mathrm{Cs}_{2} \mathrm{CO}_{3} / \mathrm{Al}\end{array}$ & $2.7(\mathrm{G})$ & $1.8(\mathrm{G})$ & \\
\hline $2014^{8}$ & $62 \Omega / \square$ & $\begin{array}{l}\text { PEDOT:PSS electrode/PEDOT:PSS (Al 4083)/ } \\
\text { TAPC/mCP:FIrpic/TPBi/LiF/Al }\end{array}$ & $24(\mathrm{G})$ & & \\
\hline $2015^{9}$ & $4200 \mathrm{~s} / \mathrm{cm}$ & $\begin{array}{c}\mathrm{ITO} / \mathrm{ZnO} / \mathrm{PEI} / \mathrm{P}-\mathrm{PPV} / \mathrm{MoO}_{\mathrm{x}} / \mathrm{PEDOT}: \mathrm{PSS} \\
\text { electrode }\end{array}$ & & $26.0(\mathrm{~W})$ & $\begin{array}{l}17.6 \\
(\mathrm{~W})\end{array}$ \\
\hline $2015^{10}$ & $123 \Omega / \square$ & $\begin{array}{c}\text { PEDOT:PSS electrode/ZnO/PEI/SY(or Merck } \\
\text { Blue)/ } \mathrm{WO}_{3} / \mathrm{PEDOT}: \mathrm{PSS} \text { electrode }\end{array}$ & $\begin{array}{c}20.1 \\
(\mathrm{Y}) / 2.5 \\
(\mathrm{~B})\end{array}$ & & \\
\hline $2015^{11}$ & $143 \Omega / \square$ & PEDOT:PSS electrode/NPB/Alq $3 / \mathrm{LiF} / \mathrm{Al}$ & $2.0(\mathrm{G})$ & & \\
\hline $2015^{12}$ & $460 \Omega / \square$ & $\begin{array}{c}\text { PEDOT:PSS electrode/PEDOT:PSS } \\
\text { HIL/SPG-01T/LiF/Al }\end{array}$ & $9.71(\mathrm{G})$ & $9.38(\mathrm{G})$ & \\
\hline $2016^{13}$ & $1265 \mathrm{~s} / \mathrm{cm}$ & PEDOT:PSS-GO/NPB/Alq $/$ /Liq/Al & $2.1(\mathrm{G})$ & & \\
\hline $2016^{14}$ & $129 \Omega / \square$ & 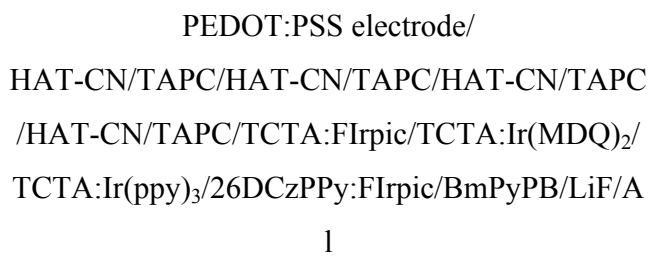 & & & $\begin{array}{l}16.2 \\
(\mathrm{~W})\end{array}$ \\
\hline $2016^{15}$ & $196 \Omega / \square$ & AgNW-PEDOT:PSS/NPB/ & $51.2(\mathrm{G})$ & & \\
\hline
\end{tabular}




\begin{tabular}{|c|c|c|c|c|c|}
\hline & & $\mathrm{CBP}: \operatorname{Ir}(\mathrm{ppy})_{3} / \mathrm{TPBi} / \mathrm{LiF} / \mathrm{Al}$ & & & \\
\hline $2017^{16}$ & & PEDOT:PSS AnoHIL/MAPbBr $/ 3$ TPBi/LiF/Al & $42(\mathrm{G})$ & & \\
\hline $2017^{17}$ & $500 \Omega / \square$ & ITO/ZnO/PEI/SY/PEDOT:PSS electrode & $3.2(\mathrm{Y})$ & & \\
\hline $2017^{18}$ & $180 \Omega / \square$ & $\begin{array}{c}\text { PEDOT:PSS/HAT-CN/NPB/HAT-CN/NPB/ } \\
\text { HAT-CN/NPB/TCTA/TCTA:FIrpic/ } \\
\text { TCTA:Ir(MDQ) } 2 \text { /TCTA:Ir(ppy) } / \\
\text { 26DCzPPy:FIrpic/BmPyPB/LiF/Al }\end{array}$ & & $26.0(\mathrm{G})$ & $\begin{array}{l}17.5 \\
(\mathrm{G})\end{array}$ \\
\hline $2017^{19}$ & $15 \Omega / \square$ & $\begin{array}{c}\text { AgNWs-PEDOT:PSS electrode/ } \\
\mathrm{MoO}_{3} / \mathrm{mCP} / \mathrm{Ir}(\mathrm{tfmppy})_{2} / \mathrm{TPBi} / \mathrm{LiF} / \mathrm{Al}\end{array}$ & $58.2(\mathrm{G})$ & & \\
\hline $2018^{20}$ & $67 \Omega / \square$ & $\begin{array}{l}\text { PEDOT:PSS/ZnO/SY or } \\
\text { White/ } \mathrm{MoO}_{3} / \mathrm{PEDOT}: \mathrm{PSS}\end{array}$ & $\begin{array}{c}2.8 \\
(\mathrm{~W}) / 2.7 \\
(\mathrm{Y})\end{array}$ & & \\
\hline $2018^{21}$ & $269 \Omega / \square$ & PEDOT:PSS electrode/NPB/Alq $3 / \mathrm{LiF} / \mathrm{Al}$ & $3.4(\mathrm{G})$ & $1.83(\mathrm{G})$ & \\
\hline \multirow{2}{*}{$2018^{22}$} & \multirow{2}{*}{$170 \Omega / \square$} & $\begin{array}{l}\text { Flat PC/PEDOT:PSS (2 Layers) } \\
\text { electrode/} / \mathrm{MoO}_{3} / \mathrm{MoO}_{3}: \mathrm{NPB} / \mathrm{NPB} / \\
\operatorname{Ir}(\mathrm{ppy})_{3}: \mathrm{CBP} / \mathrm{BPhen} / \mathrm{LiF} / \mathrm{Al}\end{array}$ & $88(\mathrm{G})$ & $82(\mathrm{G})$ & $\begin{array}{l}25 \\
(\mathrm{G})\end{array}$ \\
\hline & & $\begin{array}{c}\text { Corrugated PC/PEDOT:PSS (2 Layers) } \\
\text { electrode } / \mathrm{MoO}_{3} / \mathrm{MoO}_{3}: \mathrm{NPB} / \mathrm{NPB} / \\
\operatorname{Ir}(\mathrm{ppy})_{3}: \mathrm{CBP} / \mathrm{BPhen} / \mathrm{LiF} / \mathrm{Al}\end{array}$ & $164(\mathrm{G})$ & $144(\mathrm{G})$ & $\begin{array}{l}50 \\
(\mathrm{G})\end{array}$ \\
\hline $2018^{23}$ & $158 \Omega / \square$ & $\begin{array}{c}\text { PEDOT:PSS electrode/MeO-TPD:F }{ }_{6}-\mathrm{TCNNQ} / \\
\text { Spiro-TAD/4P-NPD:Ir(MDQ) })_{2} / 4 \mathrm{P}-\mathrm{NPD} / \mathrm{BPhen} / \\
\text { BPhen:Cs/Ag/MeO-TPD:F6-TCNNQ/Spiro-TAD/ } \\
\text { TCTA:Ir(ppy })_{3}: \operatorname{Ir}(\mathrm{dhfpy})_{2} / \mathrm{TPBi}: \operatorname{Ir}(\mathrm{ppy})_{3}: \\
\text { Ir(dhfpy })_{2} / \mathrm{TPBi} / \mathrm{BPhen}: \mathrm{Cs} / \mathrm{Al}\end{array}$ & & $26.1(\mathrm{~W})$ & $\begin{array}{l}19.6 \\
(\mathrm{~W})\end{array}$ \\
\hline $2019^{24}$ & $1467 \mathrm{~s} / \mathrm{cm}$ & $\begin{array}{l}\text { PEDOT:PSS electrode/HAT-CN/ } \\
\text { TAPC/CBP:Ir(ppy) } 3 / \mathrm{TPBi} / \mathrm{LiF} / \mathrm{Al}\end{array}$ & $26.3(\mathrm{G})$ & & \\
\hline $2019^{25}$ & $1400 \mathrm{~s} / \mathrm{cm}$ & PEDOT:PSS electrode/MAPbBr $3 / \mathrm{TPBi} / \mathrm{LiF} / \mathrm{Al}$ & $52.86(\mathrm{G})$ & & \\
\hline $2019^{26}$ & $210 \Omega / \square$ & $\begin{array}{c}\mathrm{Ag} / \mathrm{ZnO} / \mathrm{PEI} / \mathrm{Super} \text { yellow/PEDOT:PSS } \\
\text { HIL/PEDOT:PSS electrode }\end{array}$ & $10.6(\mathrm{Y})$ & $8.0(\mathrm{Y})$ & \\
\hline $2019^{27}$ & $1290 \mathrm{~s} / \mathrm{cm}$ & $\begin{array}{l}\text { PEDOT:PSS electrode/TAPC/TCTA/ } \\
\text { CBP:Ir(ppy) } 3 \text { /TmPyPB/LiF/Mg:Ag }\end{array}$ & $54.5(\mathrm{G})$ & $28.2(\mathrm{G})$ & $\begin{array}{l}15.7 \\
(\mathrm{G})\end{array}$ \\
\hline $2019^{28}$ & $71 \Omega / \square$ & $\begin{array}{l}\text { Cu-M/PEDOT:PSS electrode/PEDOT:PSS } \\
\text { HIL/PVK:CBP:Ir(mppy) })_{3} / \mathrm{TPBi} / \mathrm{LiF} / \mathrm{Al}\end{array}$ & $3.8(\mathrm{G})$ & $0.9(\mathrm{G})$ & \\
\hline $2021^{29}$ & $1583 \mathrm{~s} / \mathrm{cm}$ & $\begin{array}{l}\text { PEDOT:PSS electrode/TAPC/ } \\
\text { CBP:Ir(ppy })_{3} / \mathrm{TPBi} / \mathrm{LiF} / \mathrm{Al}\end{array}$ & $25.3(\mathrm{G})$ & & \\
\hline $2021^{30}$ & $78 \Omega / \square$ & $\begin{array}{c}\text { PEDOT:PSS electrode /PEDOT:PSS } \\
\text { HIL/TCTA/mCP:Ir(ppy)/ } / \text { TmPyPB/LiF/Al }\end{array}$ & $76.4(\mathrm{G})$ & $21.8(\mathrm{G})$ & \\
\hline
\end{tabular}




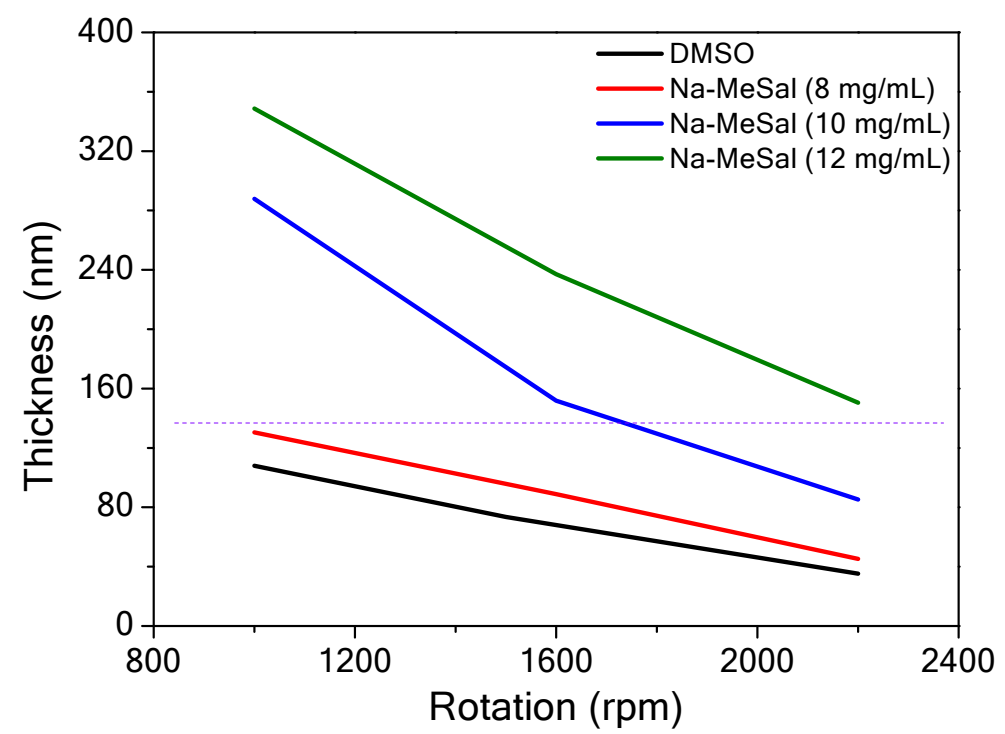

Figure S1. The rotation-dependent thickness of PEDOT:PSS TCEs with various concentrations of Na-MeSal. 

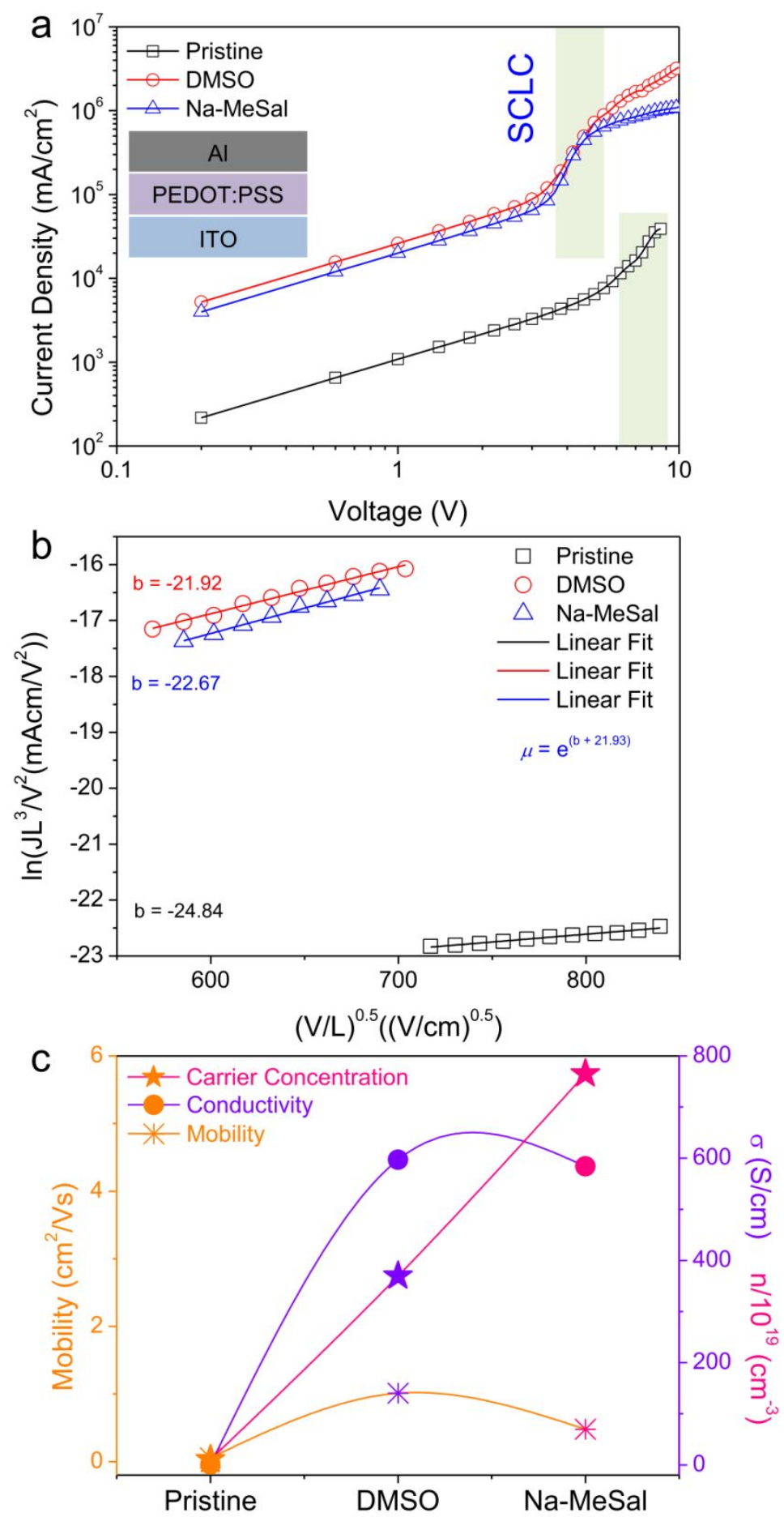

Figure S2. (a) $J-V$ curves for the hole-only devices of ITO/PEDOT:PSS (130 nm)/Al (100 $\mathrm{nm})$, (b) the plots of $\ln \left(J L^{3} / V^{2}\right)$ vs $(V / L)^{0.5}$ and the fitted intercept $b$ values according to the SCLC model equation, (c) mobility, conductivity and carrier concentration values of pristine, DMSO and Na-MeSal modified PEDOT:PSS films.

We calculated the hole mobility of PEDOT:PSS films by space-charge-limited current (SCLC) model, which can be described by the equation: 
$J=\frac{9}{8} \varepsilon \varepsilon_{0} \mu \exp \left(\gamma \sqrt{\frac{V}{L}}\right) \frac{V^{2}}{L^{3}}$

Where $J$ is the hole (electron) current density; $\varepsilon_{0}$ is the permittivity of free space $\left(8.85 \times 10^{-11}\right.$ $\mathrm{mA} \cdot \mathrm{s} / \mathrm{Vcm}$ ); $\varepsilon$ is the relative permittivity (assumed it is equal to 3 ); $\gamma$ is the field activation factor; $V$ is the voltage; $L$ is the device thickness. In order to obtain the mobility, equation (1) is changed to

$\ln \left(J L^{3} / V^{2}\right)=\gamma \sqrt{\frac{V}{L}}+\ln \left(\frac{9}{8} \varepsilon \varepsilon_{0} \mu\right)$

According to equation (2), from the plots of $\ln \left(J L^{3 / V^{2}}\right)$ vs $(V / L)^{0.5}$, where the slope is $\gamma$ and the intercept is $b=\ln \left(9 / 8 \varepsilon_{\mathrm{r}} \varepsilon_{0} \mu\right)$ of the corresponding lines on the axis of $\ln \left(J L^{3} / V^{2}\right)$. Finally, the hole mobility can be calculated from the intercept $b, \mu=2.7183^{(b+21.93)}\left(\mathrm{cm}^{2} / \mathrm{Vs}\right)$. The hole-only devices were fabricated in a device structure of ITO/PEDOT:PSS (130 nm)/Al (100 $\mathrm{nm})$. The calculated mobility for pristine, DMSO and Na-MeSal modified PEDOT:PSS films was $0.054,1.01$ and $0.48 \mathrm{~cm}^{2} / \mathrm{Vs}$, respectively. 

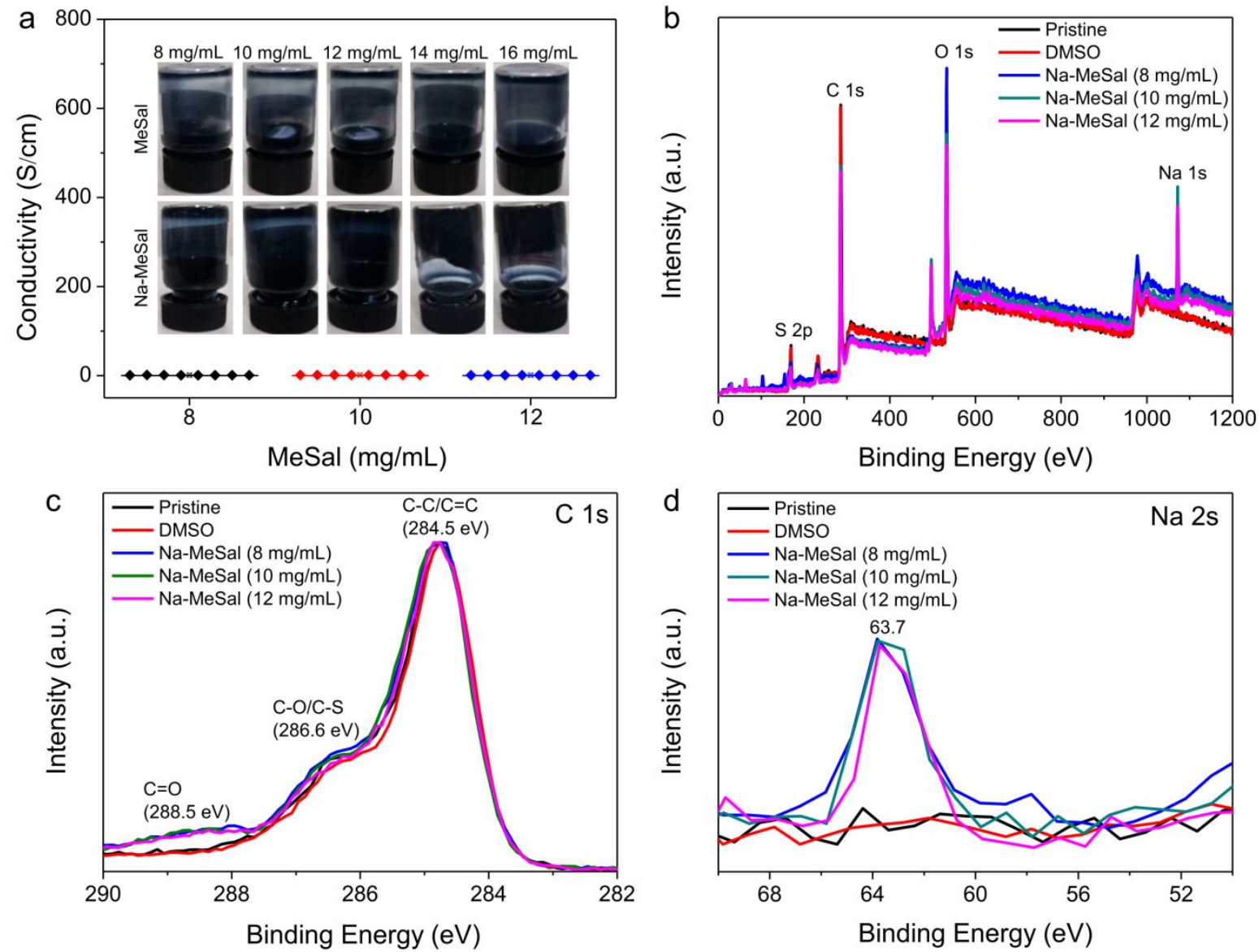

Figure S3. (a) Conductivity of PEDOT:PSS films added various concentrations of MeSal. (Inset: PEDOT:PSS solutions' picture after adding MeSal), (b) the whole survey XPS spectra, (c) C 1s, (d) Na 2S core-level spectra of pristine PEDOT:PSS film, PEDOT:PSS TCEs with DMSO and various concentrations of Na-MeSal.

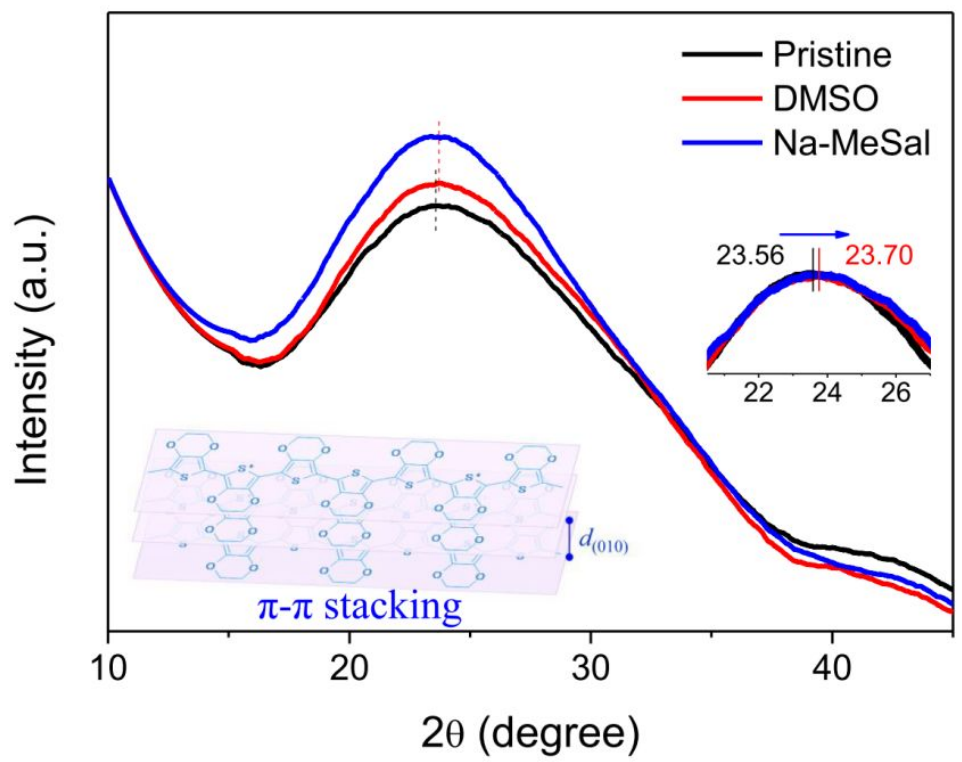

Figure S4. XRD patterns for pristine, DMSO and Na-MeSal modifed PEDOT:PSS films. 


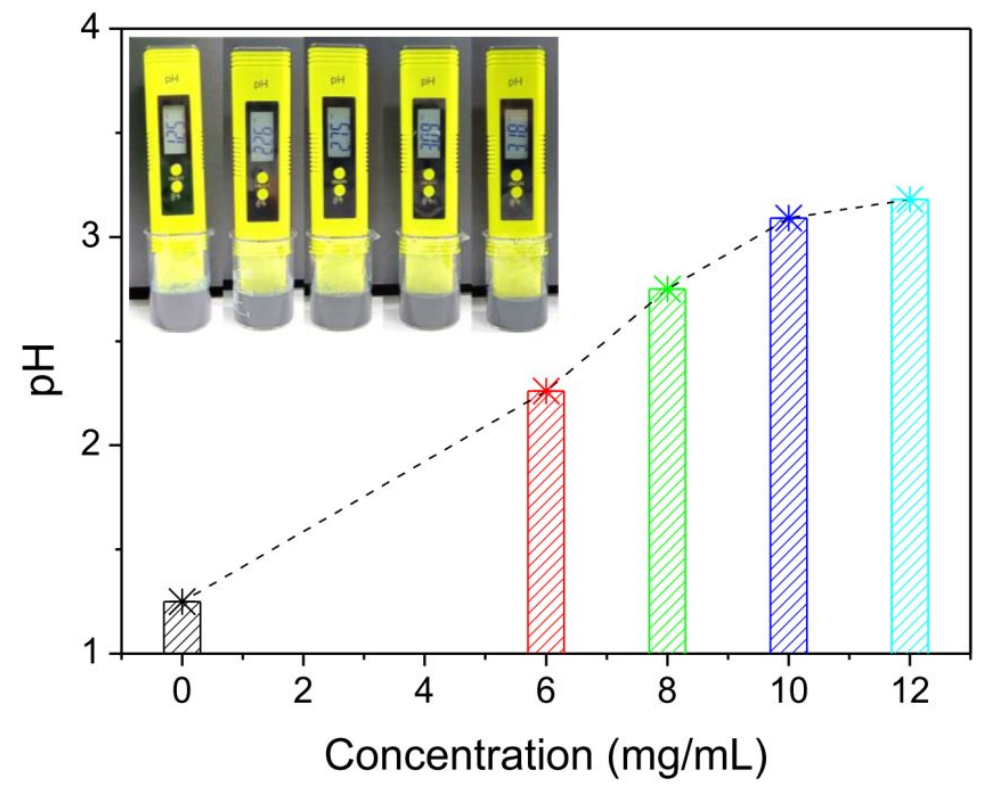

Figure S5. The $\mathrm{pH}$ values of PEDOT:PSS solutions with various concentrations of Na-MeSal salt.

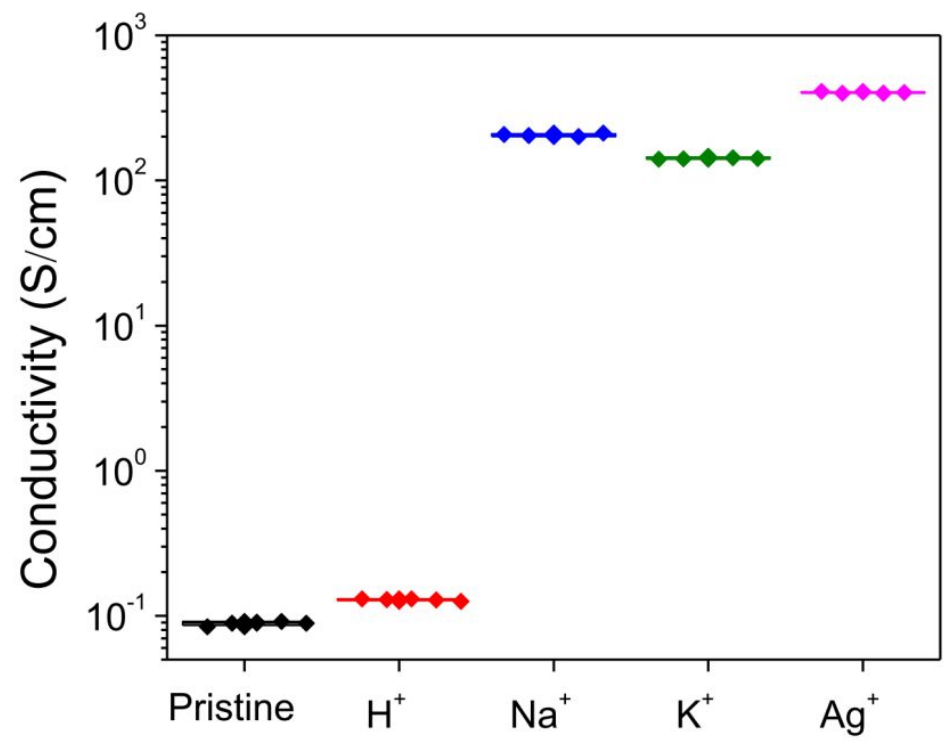

Figure S6. Conductivity of PEDOT:PSS films added benzoate, Na-benzoate, K-benzoate and Ag-benzoate. 

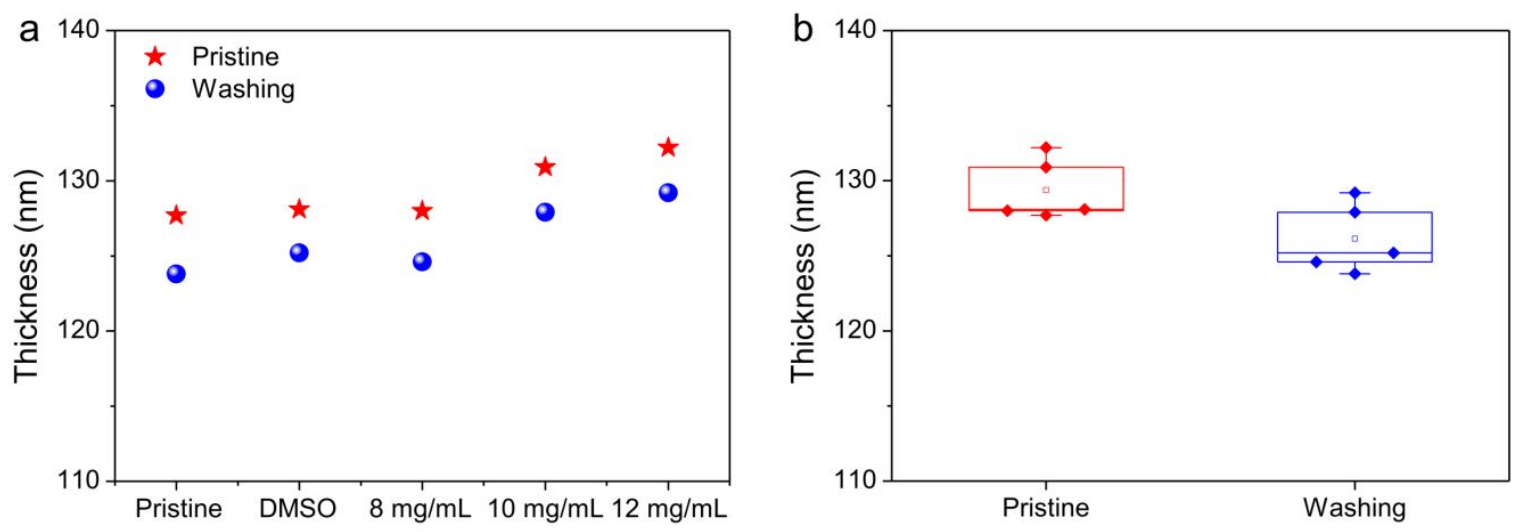

Figure S7. (a) Thickness change and the (b) average thickness of different PEDOT:PSS films before and after washing with DI water.

\section{References}

1. Kim, W. H.; Mäkinen, A. J.; Nikolov, N.; Shashidhar, R.; Kim, H.; Kafafi, Z. H., Molecular Organic Light-Emitting Diodes Using Highly Conducting Polymers as Anodes. Appl. Phys. Lett. 2002, 80, 3844-3846.

2. Fehse, K.; Walzer, K.; Leo, K.; Lövenich, W.; Elschner, A., Highly Conductive Polymer Anodes as Replacements for Inorganic Materials in High-Efficiency Organic Light-Emitting Diodes. Adv. Mater. 2007, 19, 441-444.

3. Piliego, C.; Mazzeo, M.; Cortese, B.; Cingolani, R.; Gigli, G., Organic Light Emitting Diodes with Highly Conductive Micropatterned Polymer Anodes. Org. Electron. 2008, 9, 401-406.

4. Cai, M.; Ye, Z.; Xiao, T.; Liu, R.; Chen, Y.; Mayer, R. W.; Biswas, R.; Ho, K. M.; Shinar, R.; Shinar, J., Extremely Efficient Indium-Tin-Oxide-Free Green Phosphorescent Organic Light-Emitting Diodes. Adv. Mater. 2012, 24, 4337-4342.

5. Lee, H. J.; Park, T. H.; Choi, J. H.; Song, E. H.; Shin, S. J.; Kim, H.; Choi, K. C.; Park, Y. W.; Ju, B.-K., Negative Mold Transfer Patterned Conductive Polymer Electrode for Flexible Organic Light-Emitting Diodes. Org. Electron. 2013, 14, 416-422. 
6. Zhu, X.-Z.; Han, Y.-Y.; Liu, Y.; Ruan, K.-Q.; Xu, M.-F.; Wang, Z.-K.; Jie, J.-S.; Liao, L.-S., The Application of Single-Layer Graphene Modified with Solution-Processed TiOx and PEDOT:PSS as a Transparent Conductive Anode in Organic Light-Emitting Diodes. Org. Electron. 2013, 14, 3348-3354.

7. Wu, X.; Liu, J.; Wu, D.; Zhao, Y.; Shi, X.; Wang, J.; Huang, S.; He, G., Highly Conductive and Uniform Graphene Oxide Modified PEDOT:PSS Electrodes for ITO-Free Organic Light Emitting Diodes. J. Mater. Chem. C 2014, 2, 4044-4050.

8. Ouyang, S.; Xie, Y.; Wang, D.; Zhu, D.; Xu, X.; Tan, T.; DeFranco, J.; Fong, H. H., Photolithographic Patterning of Highly Conductive PEDOT:PSS and Its Application in Organic Light-Emitting Diodes. J. Polym. Sci., Part B: Polym. Phys. 2014, 52, 1221-1226.

9. Kim, N.; Kang, H.; Lee, J. H.; Kee, S.; Lee, S. H.; Lee, K., Highly Conductive All-Plastic Electrodes Fabricated Using a Novel Chemically Controlled Transfer-Printing Method. Adv. Mater. 2015, 27, 2317-2323.

10. Zhang, M.; Hofle, S.; Czolk, J.; Mertens, A.; Colsmann, A., All-Solution Processed Transparent Organic Light Emitting Diodes. Nanoscale 2015, 7, 20009-20014.

11. Wu, X.; Liu, J.; He, G., A Highly Conductive PEDOT:PSS Film with the Dipping Treatment by Hydroiodic Acid as Anode for Organic Light Emitting Diode. Org. Electron. 2015, 22, 160-165.

12. Ha, J.; Park, J.; Ha, J.; Kim, D.; Chung, S.; Lee, C.; Hong, Y., Selectively Modulated Inkjet Printing of Highly Conductive and Transparent Foldable Polymer Electrodes for Flexible Polymer Light-Emitting Diode Applications. Org. Electron. 2015, 19, 147-156.

13. Wu, X.; Lian, L.; Yang, S.; He, G., Highly Conductive PEDOT:PSS and Graphene Oxide Hybrid Film from a Dipping Treatment with Hydroiodic Acid for Organic Light Emitting 
Diodes. J. Mater. Chem. C 2016, 4, 8528-8534.

14. Kim, J. H.; Joo, C. W.; Lee, J.; Seo, Y. K.; Han, J. W.; Oh, J. Y.; Kim, J. S.; Yu, S.; Lee, J. H.; Lee, J. I.; Yun, C.; Choi, B. H.; Kim, Y. H., Highly Conductive PEDOT:PSS Films with 1,3-Dimethyl-2-Imidazolidinone as Transparent Electrodes for Organic Light-Emitting Diodes. Macromol. Rapid Commun. 2016, 37, 1427-1433.

15. Liu, Y.-s.; Feng, J.; Ou, X.-L.; Cui, H.-f.; Xu, M.; Sun, H.-B., Ultrasmooth, Highly Conductive and Transparent PEDOT:PSS/Silver Nanowire Composite Electrode for Flexible Organic Light-Emitting Devices. Org. Electron. 2016, 31, 247-252.

16. Jeong, S.-H.; Woo, S.-H.; Han, T.-H.; Park, M.-H.; Cho, H.; Kim, Y.-H.; Cho, H.; Kim, H.; Yoo, S.; Lee, T.-W., Universal High Work Function Flexible Anode for Simplified ITO-Free Organic and Perovskite Light-Emitting Diodes with Ultra-High Efficiency. NPG Asia Mater. 2017, 9, 411-411.

17. Shu, Z.; Beckert, E.; Eberhardt, R.; Tünnermann, A., ITO-Free, Inkjet-Printed Transparent Organic Light-Emitting Diodes with a Single Inkjet-Printed Al:ZnO:PEI Interlayer for Sensing Applications. J. Mater. Chem. C 2017, 5, 11590-11597.

18. Seo, Y. K.; Joo, C. W.; Lee, J.; Han, J. W.; Cho, N. S.; Lim, K. T.; Yu, S.; Kang, M. H.; Yun, C.; Choi, B. H.; Kim, Y. H., Efficient ITO-Free Organic Light-Emitting Diodes Comprising PEDOT:PSS Transparent Electrodes Optimized with 2-Ethoxyethanol and Post Treatment. Org. Electron. 2017, 42, 348-354.

19. Wei, B.; Wu, X.; Lian, L.; Yang, S.; Dong, D.; Feng, D.; He, G., A Highly Conductive and Smooth AgNW/PEDOT:PSS Film Treated by Hot-Pressing as Electrode for Organic Light Emitting Diode. Org. Electron. 2017, 43, 182-188.

20. Kee, S.; Kim, N.; Park, B.; Kim, B. S.; Hong, S.; Lee, J. H.; Jeong, S.; Kim, A.; Jang, S. 
Y.; Lee, K., Highly Deformable and See-through Polymer Light-Emitting Diodes with All-Conducting-Polymer Electrodes. Adv. Mater. 2018, 30, 1703437.

21. Zhou, L.; Yu, M.; Chen, X.; Nie, S.; Lai, W.-Y.; Su, W.; Cui, Z.; Huang, W., Screen-Printed Poly(3,4-Ethylenedioxythiophene):Poly(Styrenesulfonate) Grids as ITO-Free Anodes for Flexible Organic Light-Emitting Diodes. Adv. Funct. Mater. 2018, 28, 1705955.

22. Hippola, C.; Kaudal, R.; Manna, E.; Xiao, T.; Peer, A.; Biswas, R.; Slafer, W. D.; Trovato, T.; Shinar, J.; Shinar, R., Enhanced Light Extraction from OLEDs Fabricated on Patterned Plastic Substrates. Adv. Opt. Mater. 2018, 6, 1701244.

23. Lee, J.; Kim, Y. H., High Performance ITO-Free White Organic Light-Emitting Diodes Using Highly Conductive PEDOT:PSS Transparent Electrodes. Synth. Met. 2018, 242, 99-102.

24. Kim, D. H.; Park, N. H.; Kim, T. W., Highly Efficient Flexible Organic Light-Emitting Devices Based on PEDOT:PSS Electrodes Doped with Highly Conductive Pyronin B. Nano Energy 2019, 65, 104027.

25. Jeong, S.-H.; Kim, H.; Park, M.-H.; Lee, Y.; Li, N.; Seo, H.-K.; Han, T.-H.; Ahn, S.; Heo, J.-M.; Kim, K. S.; Lee, T.-W., Ideal Conducting Polymer Anode for Perovskite Light-Emitting Diodes by Molecular Interaction Decoupling. Nano Energy 2019, 60, 324-331.

26. Park, J.; Yoon, H.; Kim, G.; Lee, B.; Lee, S.; Jeong, S.; Kim, T.; Seo, J.; Chung, S.; Hong, Y., Highly Customizable All Solution-Processed Polymer Light Emitting Diodes with Inkjet Printed Ag and Transfer Printed Conductive Polymer Electrodes. Adv. Funct. Mater. 2019, 29, 1902412.

27. Liu, S.; Yu, H.; Zhang, Q.; Qin, F.; Zhang, X.; Zhang, L.; Xie, W., Efficient ITO-Free 
Organic Light-Emitting Devices with Dual-Functional PSS-Rich PEDOT:PSS Electrode by Enhancing Carrier Balance. J. Mater. Chem. C 2019, 7, 5426-5432.

28. Han, J. W.; Jung, B.; Kim, D. W.; Lim, K. T.; Jeong, S.-Y.; Kim, Y. H., Transparent Conductive Hybrid Thin-Films Based on Copper-Mesh/Conductive Polymer for ITO-Free Organic Light-Emitting Diodes. Org. Electron. 2019, 73, 13-17.

29. Kang, H. S.; Kim, D. H.; Kim, T. W., Organic Light-Emitting Devices Based on Conducting Polymer Treated with Benzoic Acid. Sci Rep 2021, 11, 3885.

30. Liu, L.; Li, S.; Wu, L.; Chen, D.; Cao, K.; Duan, Y.; Chen, S., Enhanced Flexibility and Stability of PEDOT:PSS Electrodes through Interfacial Crosslinking for Flexible Organic Light-Emitting Diodes. Org. Electron. 2021, 89, 106047. 